\title{
Load-bearing characteristics of polyethylene foam: An examination of structural and compression properties
}

\author{
Eric J. Kuncir, MSBE; Roy W. Wirta, BSME; Frank L. Golbranson, MD \\ Department of Veterans Affairs Medical Center, San Diego, CA 92161
}

\begin{abstract}
Cellular foams used in shoe insoles come in a variety of structural configurations differing with respect to cell geometry. The compression of a cellular foam depends on cell reticulation and size. Examination of the compression behavior of closed cell polyethylene foams revealed distinct time-and nontime-related properties that occur during static and cyclic loading. Physical parameters were developed and determined to exactly specify pressure profiles that occur at the plantar interface. Evaluation of an interface material can be made based on peak pressures which are dependent on depth of compression, foam thickness, and physical properties of the foam. Sustained loading damages the mechanical integrity of the cellular structure such that thickness does not completely recover. Therefore, issuing several pairs of thick insoles for daily rotation is recommended for a particular foam.
\end{abstract}

Key words: biomechanics, cellular polyethylene foams, interface materials, shoe insoles.

\section{INTRODUCTION}

The use of cellular foams in the orthotics and prosthetics industries is widespread and ranges from applications as shoe insole material to prosthetic limb inserts. It is our impression that orthotic and prosthetic practitioners select interface materials, including cellular foams, in an

Address all correspondence and requests for reprints to: Roy W. Wirta, 5570 Rab Street, La Mesa, CA 92042.

This work was supported by a grant entitled "Foot Interface Pressure Study," from the Department of Veterans Affairs Rehabilitation Research and Development Service and was conducted at the DVA Medical Center, San Diego, California. arbitrary fashion based on availability and personal knowledge. Contributing to the arbitrariness of material selection is a lack of published information on detailed mechanical properties of cellular foams. In order to provide a basis for a more complete understanding of cellular foam properties, our investigative efforts have been directed toward an examination of the fundamental properties of cellular foams. Specifically, we have studied the structural and compression properties of cellular foams. An application of these findings is presented in an examination of the cushioning characteristics of some polyethylene foams. This discussion is relevant to orthotists and prosthetists because it addresses an overview of the properties of cellular foams, the knowledge of which may be useful in the determination of the function of a particular foam material in loadbearing applications. It should be noted at the outset that we are not a testing laboratory and that the extent of material testing was limited to support of a grant concerned with the prevention and treatment of diabetic plantar ulcers.

\section{STRUCTURE OF FOAMS}

Cellular polyethylene foams such as Aliplast, Plastazote, and Pelite are best described as a mass of bubbles composed of a plastic and a gas phase. The polymer is distributed in the walls of the bubbles and the lines where the bubbles intersect (1). The bubbles are referred to as cells, the lines of intersection are called ribs or strands, and the walls are called windows. Cell windows which consist of polymer material are called nonreticulated cell walls; windows with no structural wall are called reticulated cell 
Journal of Rehabilitation Research and Development Vol. 27 No. 3 Summer 1990

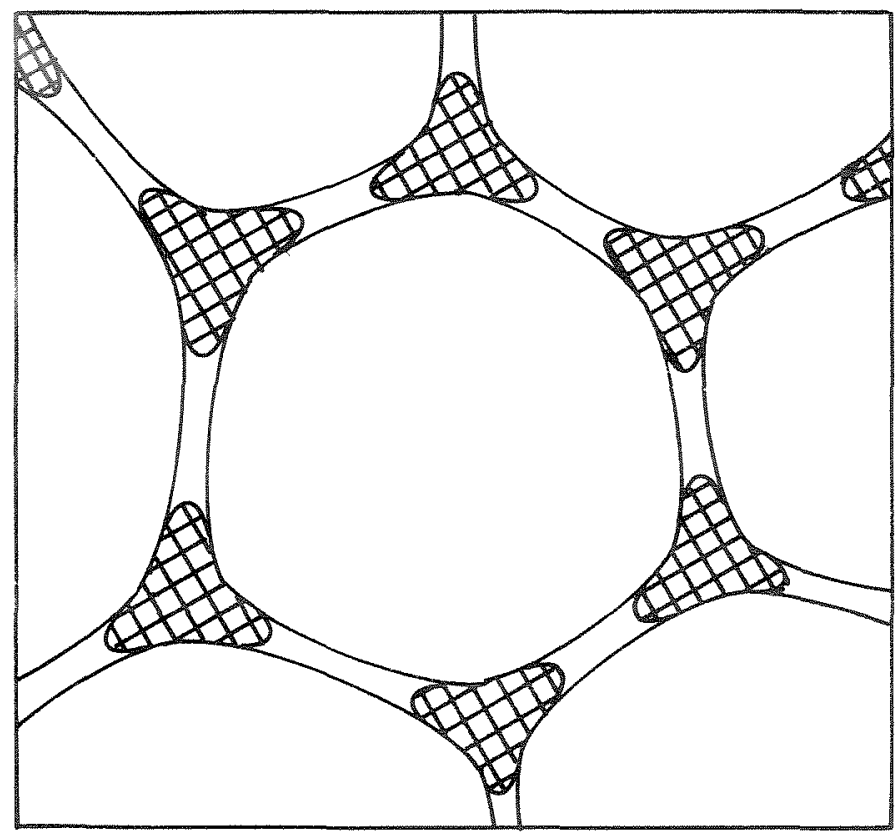

Figure 1a.

A stylized diagram of an open cell structure illustrating cell strands and walls.

walls. Depending on the configuration of this two-phase gas/solid system and on the synthetic material used, cellular plastics exhibit a wide range of mechanical properties. Hence, for a particular polymer, these properties are controlled by the structural features of the cellular material. In general, two major descriptions are offered to characterize structural features of cellular materials. An open cell material is one which has open windows leaving many cells interconnected in such a manner that gas may pass from one cell to another. Alternatively, closed celled materials are made up of discrete cells through which gases do not pass freely. Stylized diagrams of open and closed cell structures are presented in Figure 1a and Figure 1b. Note that for the open cell structure, the bulk of the polymer is in the strands. In the closed cell structure, the polymer material is also distributed in the walls, thus preventing gas movement. The open and closed celled classifications of cell structure are important because a knowledge of cell geometry will be desirable in an analysis of physical properties.

\section{PHYSICAL PROPERTIES}

One major dependence of mechanical properties on structural variables is demonstrated by compressive loading data. A physical test of the mechanical behavior of a material can be done by continuously measuring the force

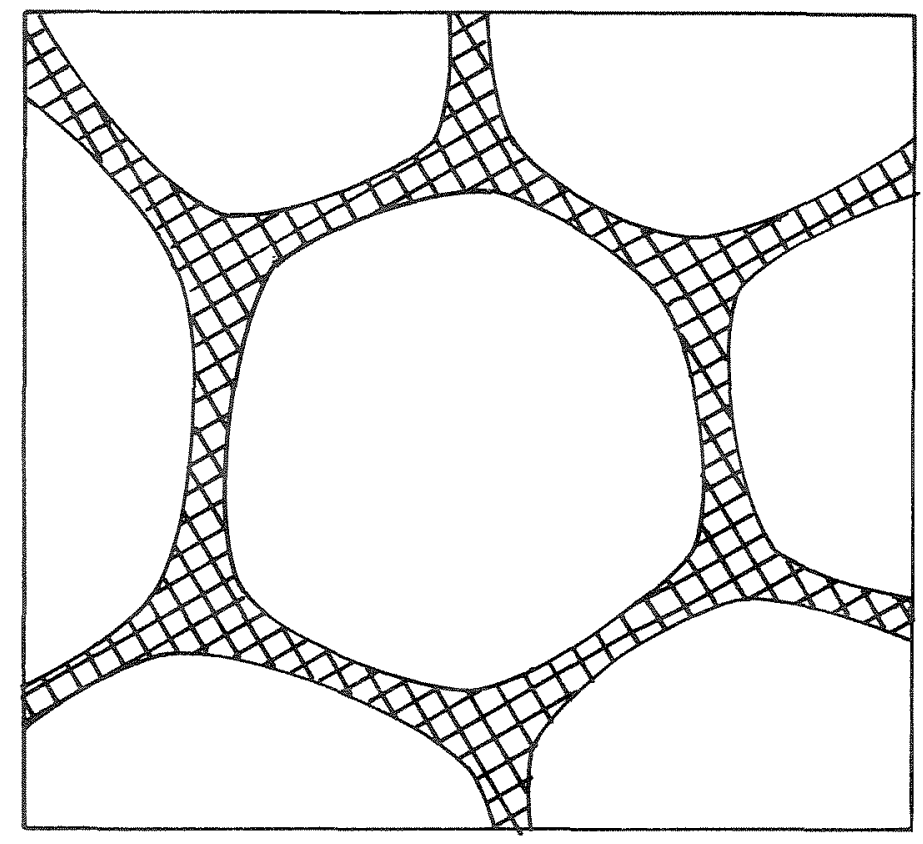

Figure 1b.

A stylized diagram of a closed cell structure illustrating cell strands and walls. A closed cell structure prevents gas movement.

required to develop a degree of compression. This information is useful because it aids in an evaluation of a foam's response under load-bearing conditions. Results of compression data for polyethylene foam obtained by Skochdopole (2) are illustrated in Figure 2 which is a plot of compressive load versus percent compression for polyethylene foams of increasing open cell character. The method of compression testing described above was used to obtain compressive load data; load indicating force per unit area. These data show that compressive load of polyethylene foam increases as the fraction of open cells decreases. Consequently, it can be concluded that cell geometry is important in determining the compression characteristics of cellular foams. That is, an interpretation of the Skochdopole results suggests that the distribution of foam material in either the cell ribs or the cell walls influences the compression behavior of the cellular foam. Since this is the case, a consideration of cell geometry is required in the proper use of these materials in load-bearing applications (i.e., as in foot-orthotic devices.)

In order to clarify this result, a model of cellular foam consisting of two reactive elements in parallel was proposed by Skochdopole. In this model, the reactive elements are the cell walls and the trapped gas, as shown in Figure 3. From the curve produced for compression of the cell walls, it is observed that at low compressions the resistance of the cell wall dominates. This region then levels off when 


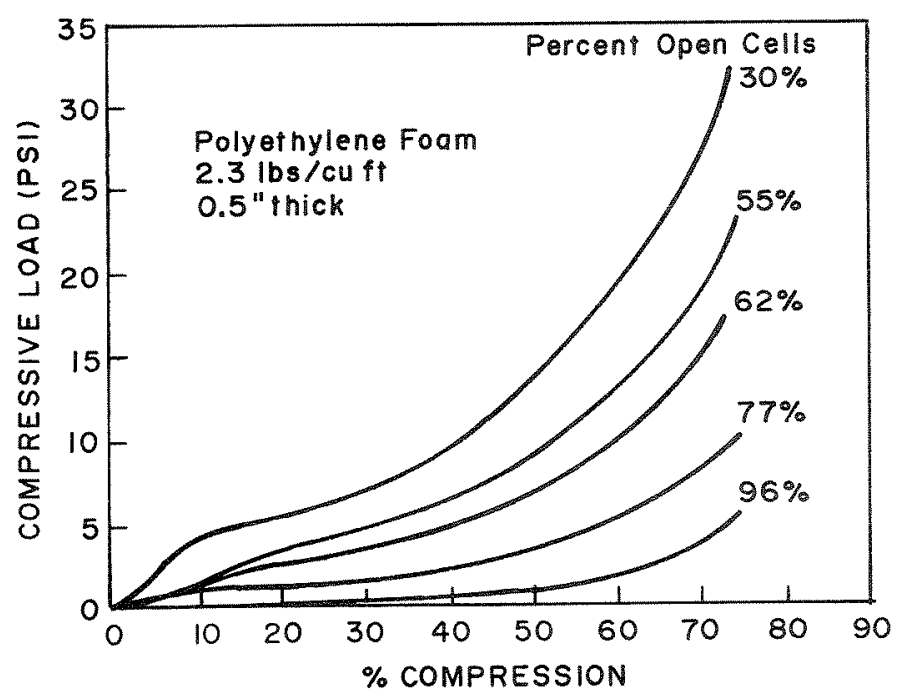

Figure 2.

Compressive load versus compression for different fraction open cells.

the cell walls reach a critical buckling stress and this behavior continues until the matrix is collapsed. At this point, additional loading does not further compress the material. Alternatively, compression of the closed cell element shows that there is a rise in pressure of the trapped gas. Based on this cellular foam model, compression of a foam sample must represent the resultant compressive resistance produced by both elements. In Figure 2, the high compressive load upon initial compression for a foam with few open cells may be interpreted as a domination of the compression force by the cell walls. That is, when there is a small fraction of open cells, the compression force is distributed over a larger number of cell walls and ribs, thereby increasing the compressive resistance. At larger degrees of compression, the data presented in Figure 2 indicates that compressive load increases as the fraction of open cells decreases. The cell geometry model predicts this behavior because gas trapped in closed cells begins to offer increased amounts of compressive resistance as a cellular foam is compressed. This implies that foams of increased open cell character must provide less resistance to escape of gases, which explains the reduction in compression resistance as open cell character increases.

Having determined the effect of reticulation on compression behavior, we can now address the influence of cell size. Knowledge of the influence of cell size on foam compression behavior is important because it is often necessary to compare the loading characteristics of two materials of similar degrees of reticulation. An example would be compression of two closed cell foam samples of similar density which differ slightly with respect to cell size. The larger cell foam illustrated in Figure 4 a has fewer ribs and walls that undergo compression compared to the small cell foam shown in Figure $\mathbf{4 b}$. Hence, if the compression of foam is interpreted as a buckling of cell walls and the intercellular movement of gases, it would appear that the effect of cell size would be significant. The dependence of compression load upon cell size for polyethylene foam is shown in Figure 5, reproduced from Skochdopole. Compressive load is plotted versus percent compression for two polyethylene foam samples of similar density which differ considerably with respect to cell size. There is a noted difference in compression behavior at larger compressions. Viewing the ribs of the cellular structure as columns in compression, the columns are shorter in the small cell structure than in the larger cell structure. One could argue that a tall column would buckle sooner under a given load than a short column. This, coupled with higher resistance to intercellular gas flow in a small cell structure, explains the differences between curves $\mathrm{A}$ and $\mathrm{B}$ in Figure $\mathbf{5}$ at larger compressions.

In summary, based on evidence provided by compression data for polyethylene foam, it can be concluded that the influence of cell geometry on the mechanical properties of cellular foams is significant. Specifically, increased compression strength is acquired as the cell diameter decreases. In addition, decreasing the fraction of open cells increases the required force for a given degree of compression. An examination of compression data therefore reveals a relation between structural variables and mechanical
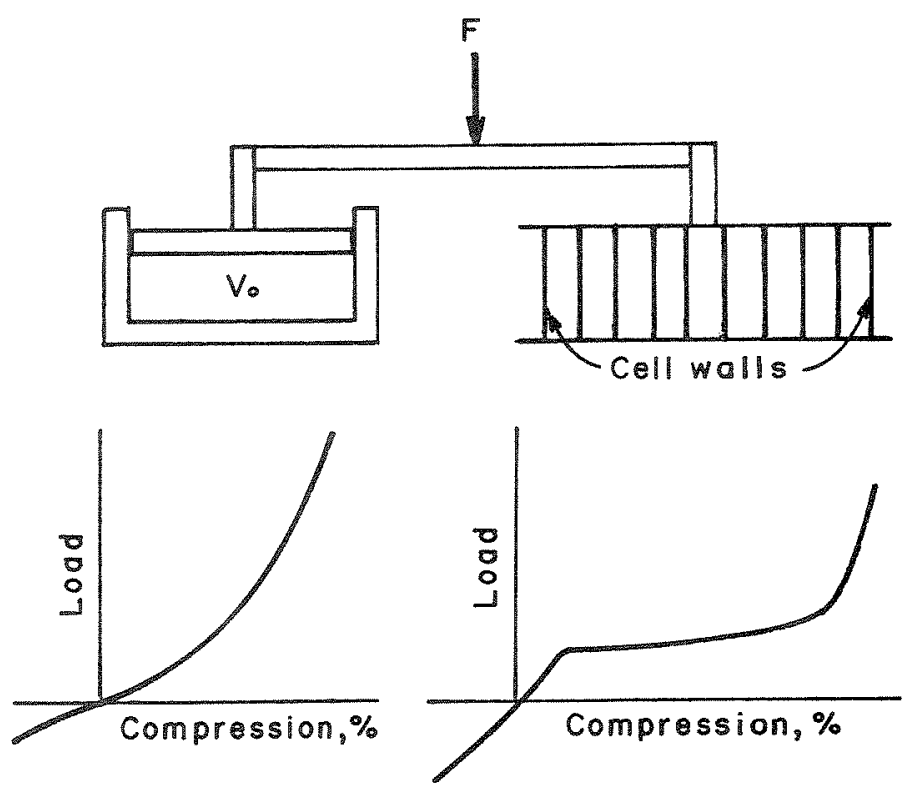

Figure 3.

Mechanical model for polyethylene foam. 


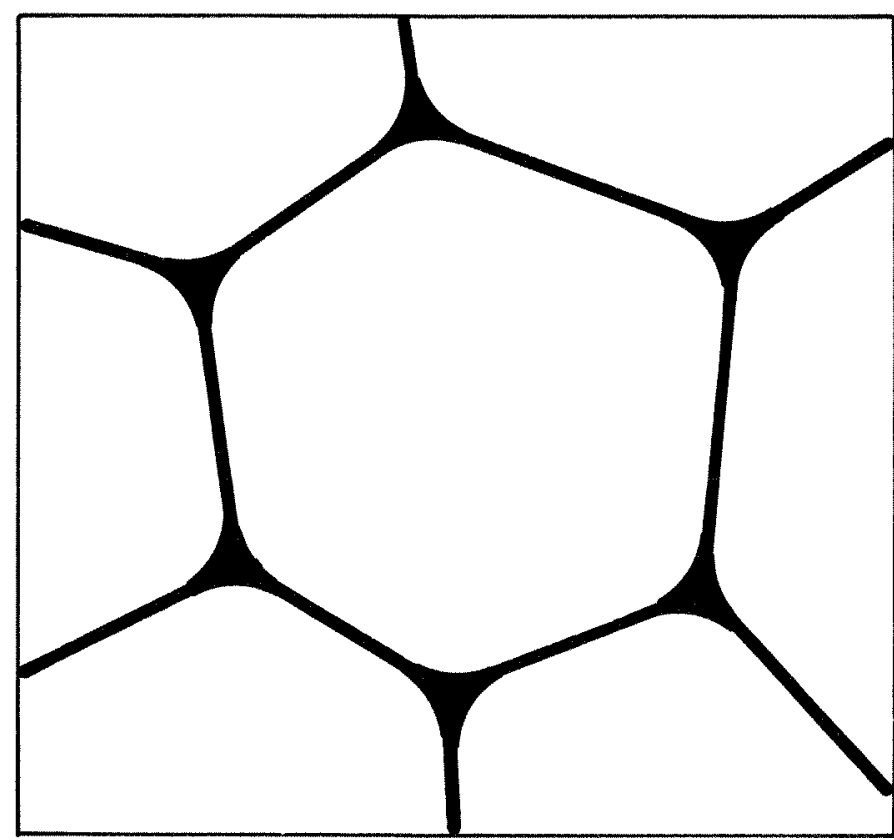

Figure 4a.

A closed cell foam sample of large cells, differing slightly with respect to cell size, but of similar density to the cells shown in Figure $\mathbf{4 b}$. Cell size determines the relative number of ribs and walls.

properties for cellular foams: mechanical properties are dependent on the proportion of open cells and on cell size.

With this background information, we can now consider the use of foams under load-bearing conditions with special consideration given to closed cell foams. Closed cell polyethylene foam materials exhibit both time-related and nontime-related properties under load-bearing condi-

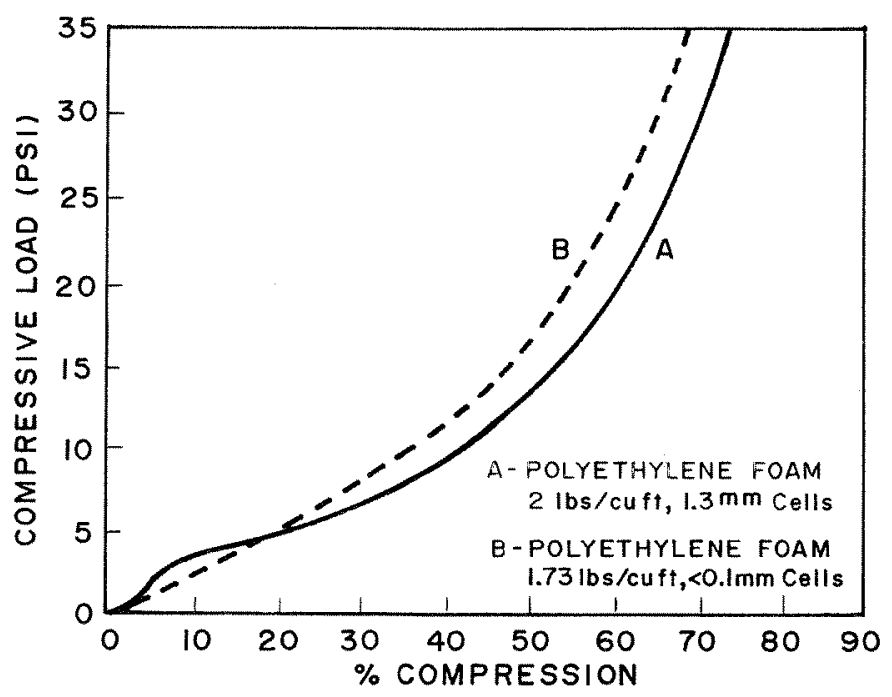

Figure 5.

Compressive load versus compression for different cell sizes.

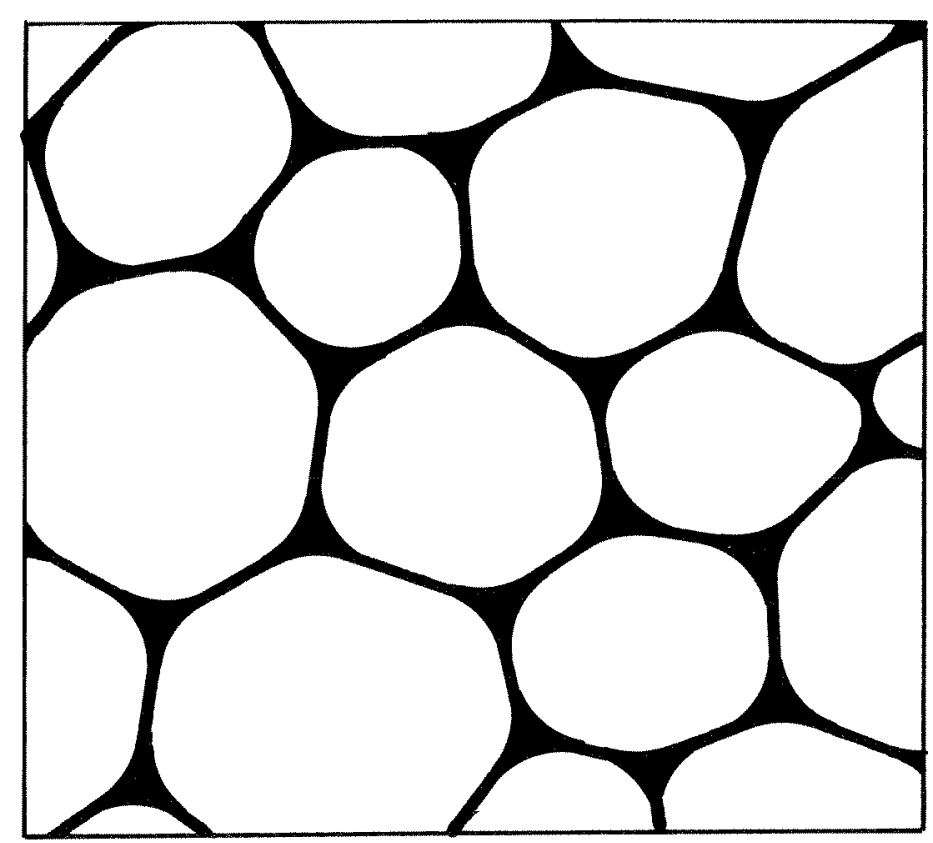

Figure $4 b$.

A closed cell foam sample of small cells, differing slightly with respect to cell size, but of similar density to the cells shown in Figure 4a. Cell size determines the relative number of ribs and walls.

tions. The nontime-related properties happen under rapid cyclic loading conditions as may occur during a gait cycle. The time-related properties happen when a load is sustained either as a static load or an extended period of cyclic loading. Under short-duration cyclic loading conditions, the thickness of the material recovers instantly and totally on removal of the load. Under static or prolonged cyclic loading, the material does not return instantly to original thickness on removal of the load. The reason for the latter phenomenon is that, while the structure may be closed cell, the cell walls are not totally impermeable to the flow of gases. Under sustained load, gases are squeezed out; when the load is removed, gases are drawn back into the cells. The recovery is a result of potential energy stored in the cellular matrix.

In particular, nontime-related characteristics resemble a nonlinear spring in load-thickness relations. Figure 6, which is a typical thickness versus load relation, illustrates this point. This nontime-related characteristic was determined with a manually operated device which plotted the relation between thickness and the applied force (i.e., pressure). The sample was placed between the jaws of the device, force was applied by a manually operated lever, and an attached pen traced the resulting curve. During loading, energy is stored in both the compression of the gases in the cells and in elastic deformation of the plastic. 


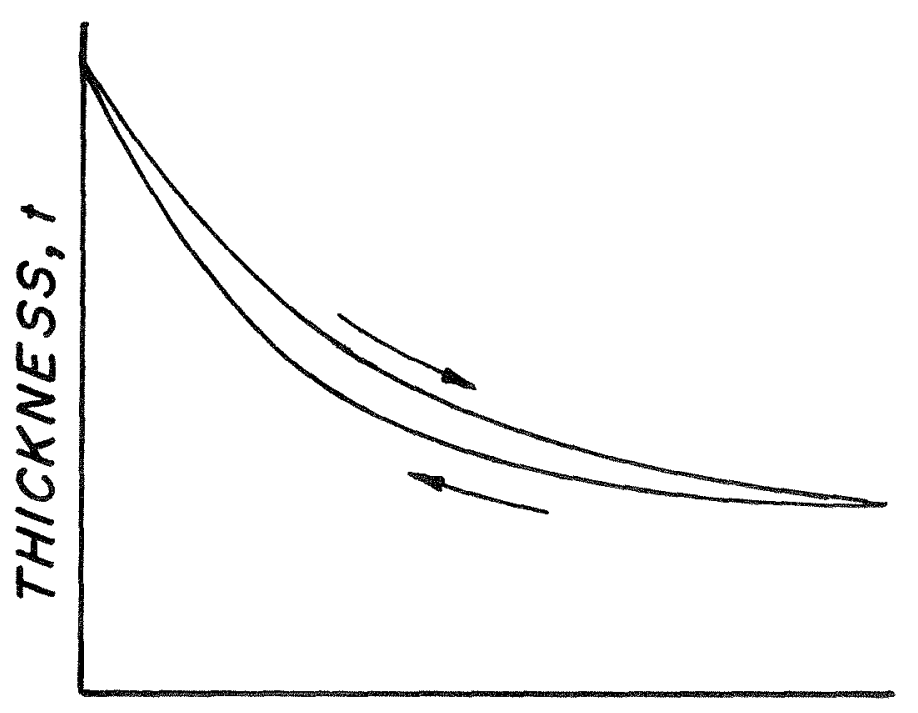

\section{PRESSURE, P}

Figure 6.

Typical relation of thickness as a function of applied pressure.

When the load is released, the stored energy restores the thickness of the material to its original value. A small amount of hysteresis may be evident as represented by the loading and unloading paths shown in Figure 6.

The relation between the thickness and load may be characterized adequately for discussion purposes by the following approximation:

$$
t=\left(t_{0}-t_{c}\right) e^{-a p}+t_{c}
$$

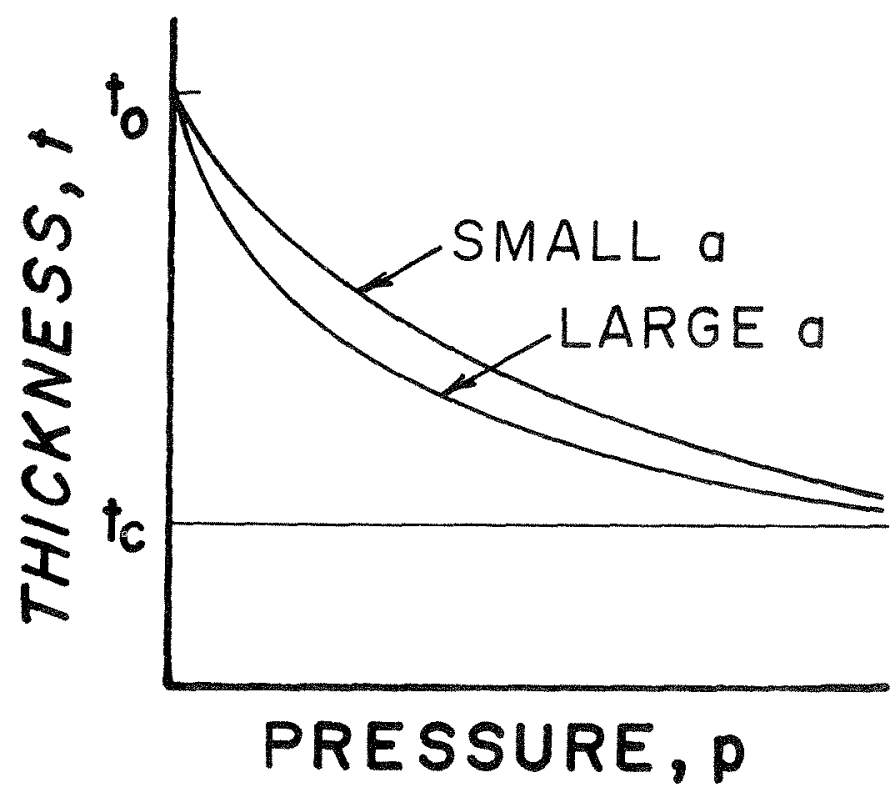

where $t$ is the thickness at any load $p, t_{0}$ is the unloaded thickness, $t_{c}$ is the thickness at large loads where the curve flattens out and approaches a limit, and $a$ represents the compliance factor. The compliance factor may vary from one material to another as well as the relation of $t_{c}$ to $t_{0}$. If the relation of $t_{c}$ to $t_{o}$ is given as $r=t_{c} / t_{o}$, then the term $r$ represents the compressibility factor. Substituting $r$ into the above expression results in the following:

$$
\mathrm{t}=\mathrm{t}_{0}(1-r) \mathrm{e}^{-a \mathrm{p}}+r \mathrm{t}_{\mathrm{o}}
$$

The form of this expression shows that $a$ and $r$ are independent parameters.

The effects of different compliance and compressibility factors on the thickness-load relations are illustrated in Figures $7 \mathbf{a}$ and $7 \mathbf{b}$. The compliance factor characterizes the rapidity at which the thickness changes with load to reach the limit $t_{c}$ as shown in Figure 7a. The compressibility factor, on the other hand, characterizes the fraction of the unloaded thickness at which the $t_{c}$ reaches a limit as shown in Figure $\mathbf{7 b}$. Values of compliance factor and compressibility factor are listed in Table 1 for selected commercially available polyethylene cellular foams. The work required in a compression from 0 to $40 \mathrm{psi}$ ( $276 \mathrm{kPa}$ ) indicating the potential energy required to overcome both gas compression and cell wall compression is also listed. Values listed in Table 1 are presented graphically in Figure 8 and Figure 9. The compliance factor is plotted versus compressibility factor for 20 percent compression and 30 percent compression in Figure $\mathbf{8}$ and work data are plotted in Figure 9. The reader is invited to explore these graphs

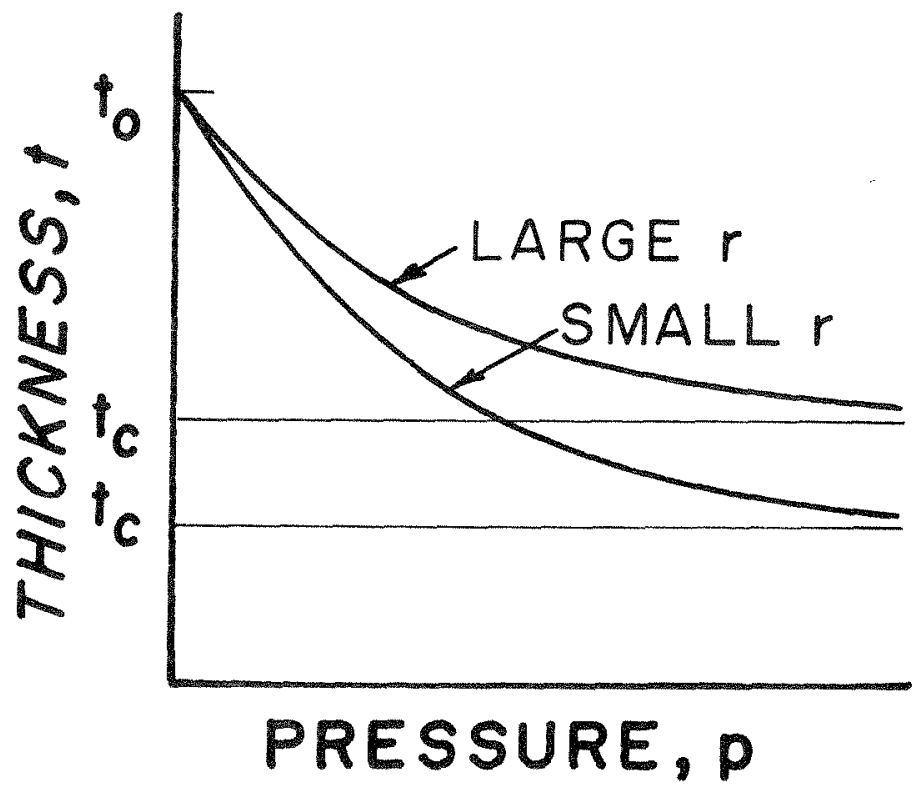

Figure 7.

Effects of two properties in the applied pressure/thickness relation. a) Compliance factor $a$; and, b) compressibility factor $r$. 
Journal of Rehabilitation Research and Development Vol. 27 No. 3 Summer 1990

Table 1.

Characteristics of selected polyethylene foams. All values are normalized to one inch $(2.54 \mathrm{~cm})$ cube where $a=$ compliance factor, $r=$ compressibility factor, and $w=$ work (inch-pound) for compression from 0 to $40 \mathrm{psi}(276 \mathrm{kPa})$.

\begin{tabular}{llcc} 
& & $a$ & $r$ \\
1. Evazote & .0519 & .222 & 22.0 \\
2. Soft Pelite & .0414 & .190 & 23.4 \\
3. Medium Pelite & .0483 & .394 & 26.5 \\
4. Firm Pelite & .0292 & .420 & 30.5 \\
5. Aliplast 4E & .0427 & .249 & 24.4 \\
6. Aliplast 6A & .0317 & .377 & 28.1 \\
7. Med. Plastazote & .0443 & .271 & 24.3 \\
8. Firm Plastazote & .0472 & .468 & 28.3 \\
9. Med. Dermaplast & .0317 & .237 & 26.8 \\
10. Firm Dermaplast & .0514 & .479 & 28.0 \\
\hline
\end{tabular}

by first examining the effects of different compliance factors at a given compressibility factor. Note that the material with the larger compliance factor requires less pressure to compress the material by a given percentage. Next, examine the effects of different compressibility factors at a given compliance factor. Note that materials with a larger compressibility factor require more pressure to compress the material by a given percentage. Finally, note that certain combinations of compliance and compressibility factors require the same applied pressure to produce the same percent compression. A similar explanation may be followed regarding work relating to different combinations of compliance and compressibility factors, as shown in Figure 9.

In order to more clearly specify the nontime-related compression behavior of cellular foams, a comparison of the compression behavior of two polyethylene foams with the adiabatic compression behavior of air is presented in Figure 10. This graph suggests that under rapid loading

\section{$20 \%$ COMPRESSION}

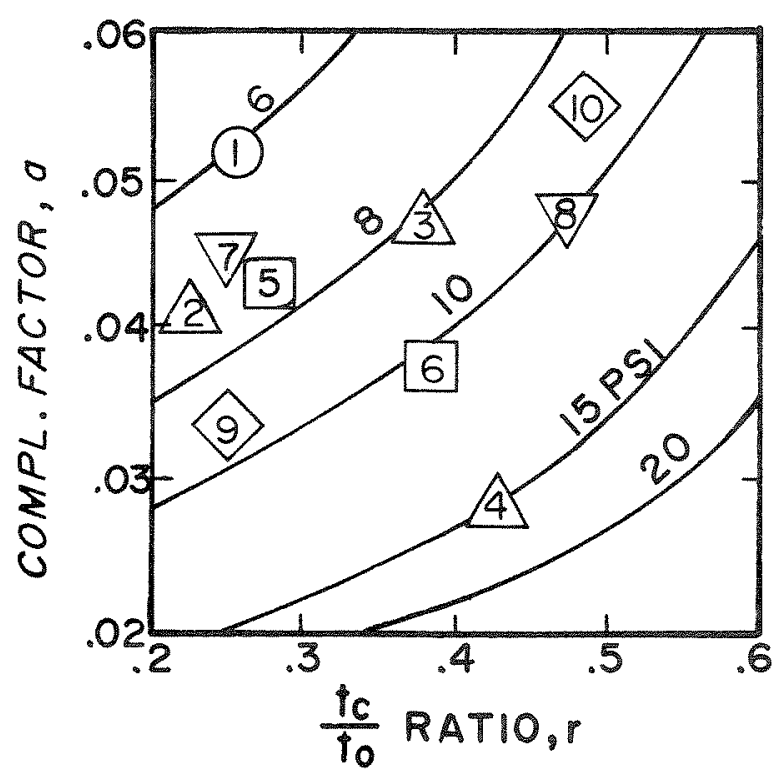

1. EVAZOTE

2. PELITE, SOFT

3. PELITE, MED.

4. PELITE, FIRM

5. ALIPLAST 4E

\section{$30 \%$ COMPRESSION}

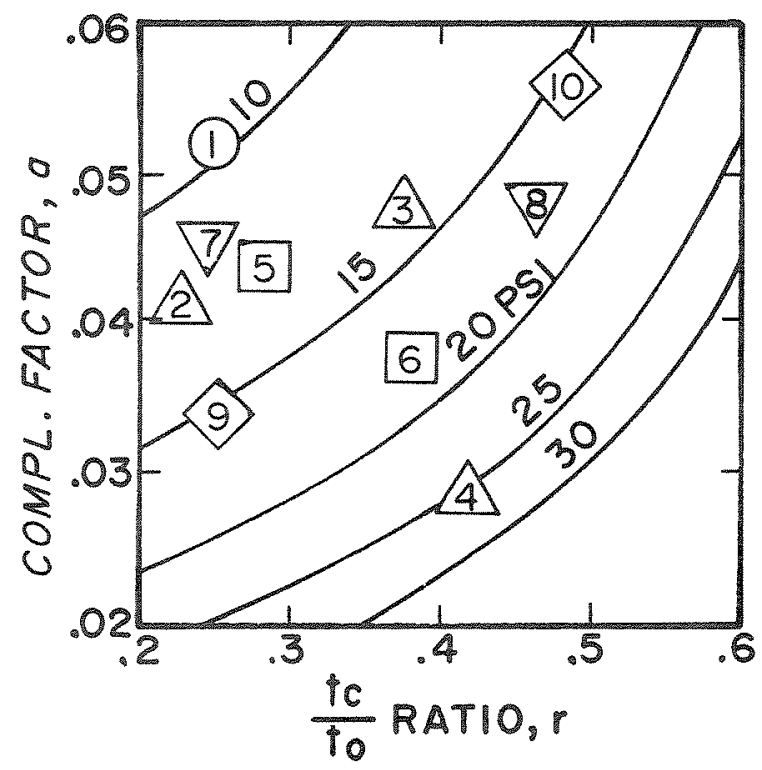
6. ALIPLAST 6A
7. PLASTAZOTE, MED,
8.PLASTAZOTE, FIRM
9.DERMAPLAST, MED.
10. DERMAPLAST, FIRM

Figure 8.

Distribution of 10 different materials by compliance and compressibility factors showing pressure parameters required to compress the materials 20 and 30 percent. 


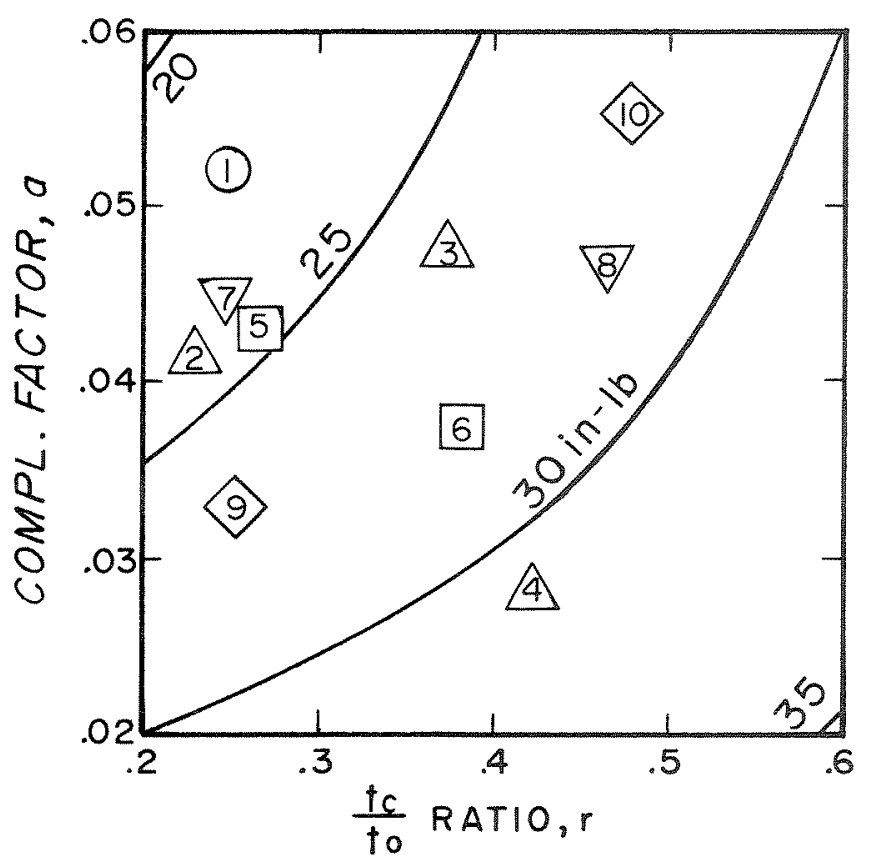

1. EVAZOTE 2.PELITE, SOF T

6. ALIPLAST 6A 3.PELITE, MED. 4.PELITE, FIRM 5.ALIPLAST 4E

7. PLASTAZOTE, MED. 8. PLASTAZOTE, FIRM 9. DERMAPLAST, MED. 10. DERMAPLAST, FIRM

Figure 9.

Distribution of 10 different materials by compliance and compressibility factors showing work parameters (in-lb) required to compress a 1-inch $(2.54 \mathrm{~cm})$ cube of material with 40 psi $(276 \mathrm{kPa})$ pressure.

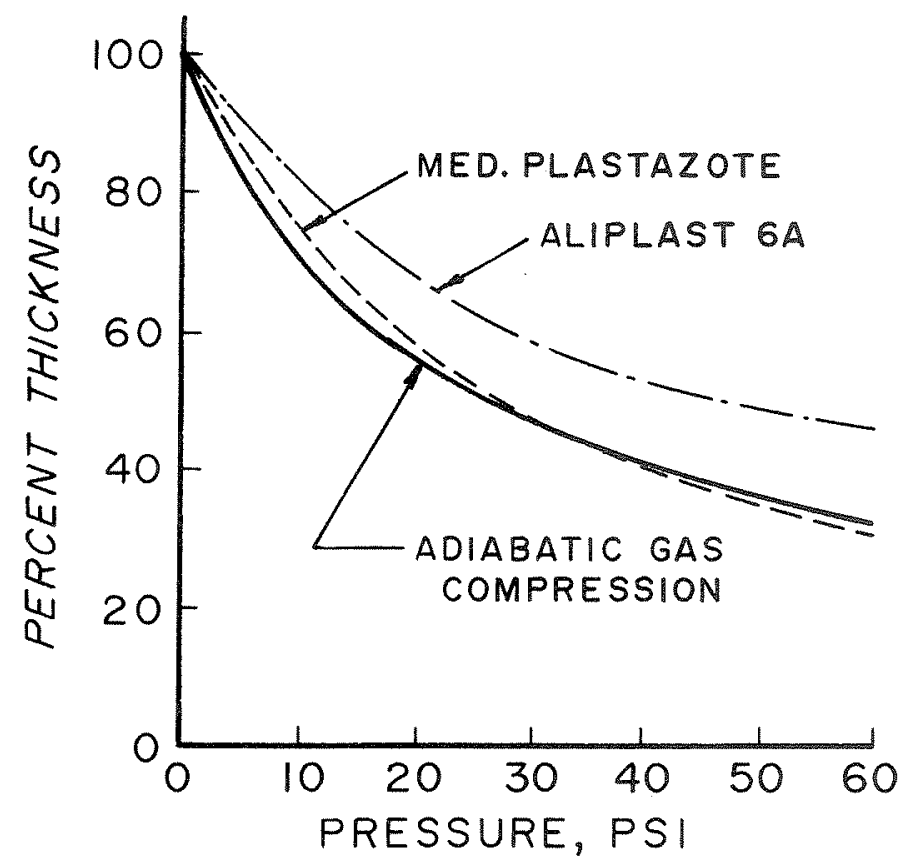

Figure 10.

Comparison of compression behavior of two polyethylene foams with adiabatic compression behavior of air. the plastic foam materials tend to conform to the gas law. That is, the gas provides the major factor in the relation between pressure and thickness. Presumably, differences in the compression behavior of the foams and the gas are due to the resistance of the plastic foam matrix to elastic deformation. The difference in the compression behavior for Plastazote and Aliplast in Figure 10 therefore indicates a dependence of elastic deformation on the value of the compliance factor, $a$. Work per one-inch $(2.54 \mathrm{~cm})$ cube of gas pressure in the compression of the gas from 0 to 40 psi $(276 \mathrm{kPa})$ is $23.6 \mathrm{in} \cdot \mathrm{lb}(2.6 \mathrm{~J})$. This is less than for any of the foam materials indicating some degree of lost work. That is, some energy is not returned but is converted instead into heat and structural damage.

The abstractiveness of compliance and compressibility factors can be reduced through graphical illustrations which demonstrate their effects on pressure profiles. Picture a spherical surface being pushed into a plastic foam and then imagine the resulting pressure profile at that surface. To help visualize this setting, refer to Figure $\mathbf{1 1}$ and Figure 12. To demonstrate the effects of different materials, the mathematical equations given earlier were used to calculate pressure profiles. In each case, a spherical surface of 3-inch radius $(7.62 \mathrm{~cm})$ is shown pushed 0.255 and 0.125 inches $(0.57$ and $0.32 \mathrm{~cm})$ into plastic foams 0.375 and 0.50 inch $(0.95$ and $3.04 \mathrm{~cm})$ thick. Aliplast $6 \mathrm{~A}$ and Aliplast $4 \mathrm{E}$, in Figures 11 and 12 respectively, were selected only for illustrative purposes because of differing compliance and compressibility factors. For the illustration, the following factors were used: Aliplast 6A, $a=0.037$ and $r=0.337$; Aliplast 4E, $a=0.043$ and $r=0.25$. As anticipated, the resulting peak pressures are dependent upon the physical properties of the plastic foams, their thicknesses, and depth of compression. These diagrams demonstrate the critical role that choices in material and thickness play regarding interface pressures. Within the realistic limits presented in Figures $\mathbf{1 1}$ and $\mathbf{1 2}$, the peak pressures range from a minimum of $10 \mathrm{psi}(69 \mathrm{kPa})$ to a maximum of $90 \mathrm{psi}(620$ $\mathrm{kPa}$ ). The magnitudes of peak pressures under varying conditions infer several aspects to patient care. First, the relative sensitivity to depth of compression suggests that a small reduction in depth of compression may result in a substantial reduction in pressure. Second, choices in physical properties and thickness of the plastic foam can significantly affect the resulting pressures under like conditions. Third, cyclic loading under high pressure conditions will squeeze out gases more quickly from the cellular structure than under lower pressure, leading to reduced cushioning in a shorter interval of use. That is why foam plastics in this category tend to mold to the contour of the plantar surface during extended use. Conspicuous by its 

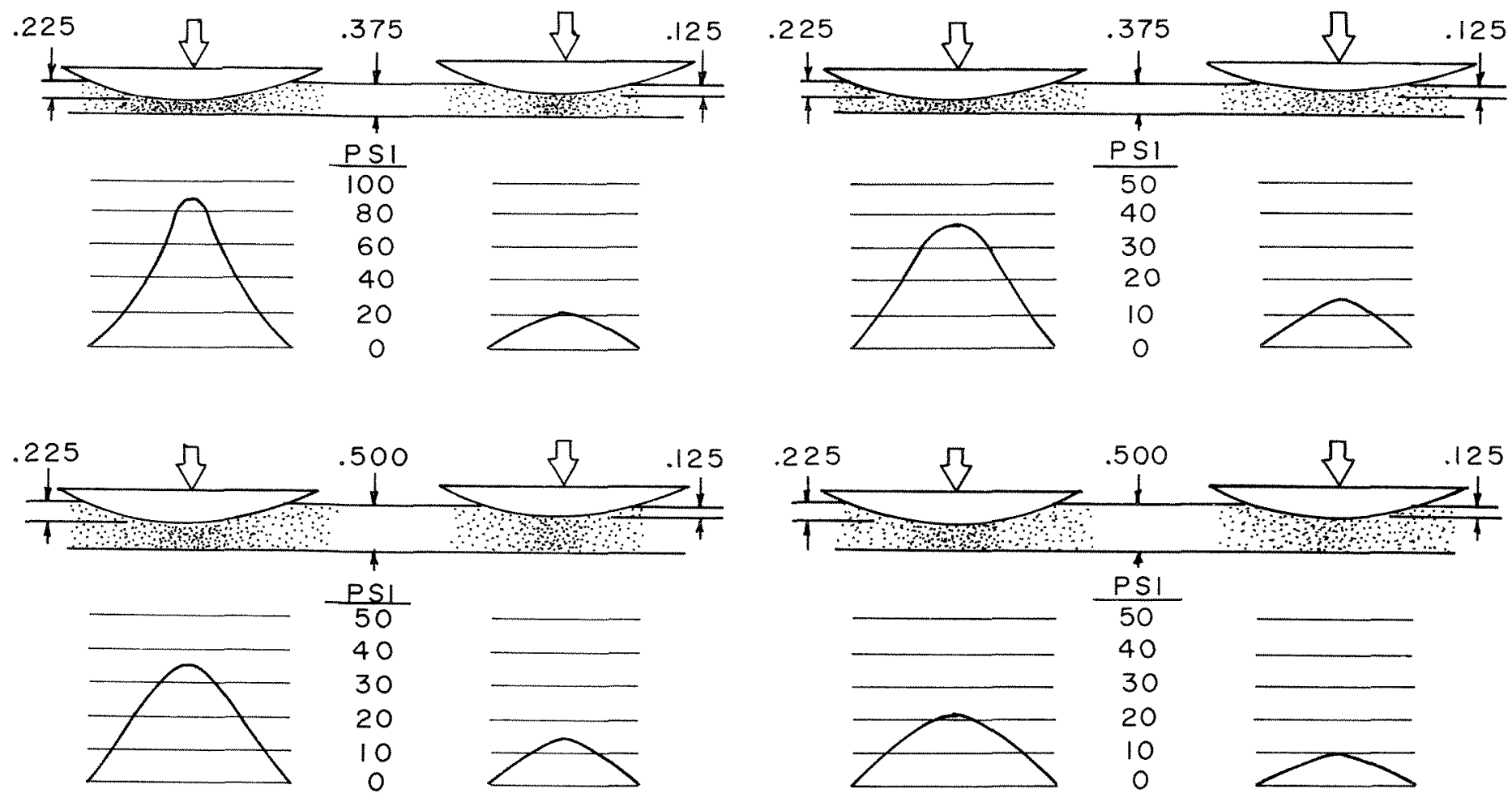

Figure 11.

Pressure profiles at the interface of a 3 -inch $(7.62 \mathrm{~cm})$ radius spherical surface for two depths of compression for three-eighths and onehalf inch $(0.95$ and $1.27 \mathrm{~cm})$ thick Aliplast $6 \mathrm{~A}$.

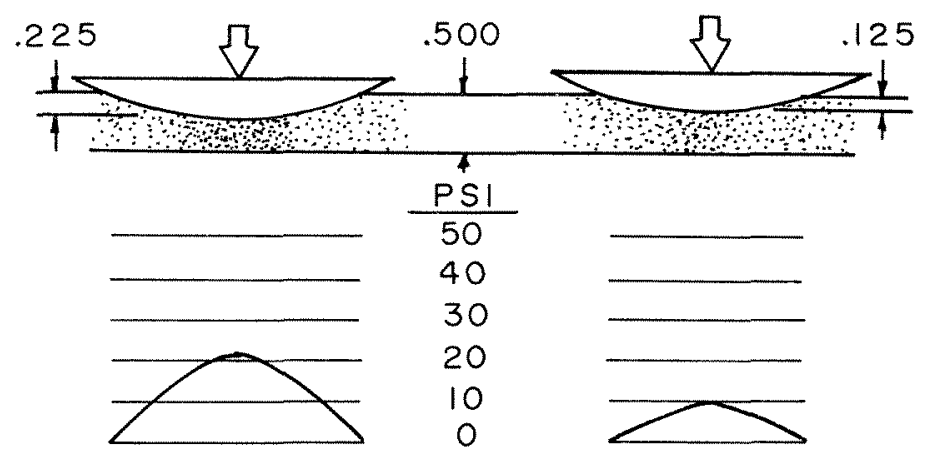

Figure 12.

Pressure profiles at the interface of a 3 -inch $(7.62 \mathrm{~cm})$ radius spherical surface for two depths of compression for three-eighths and onehalf inch $(0.95$ and $1.27 \mathrm{~cm})$ thick Aliplast $4 \mathrm{E}$.

absence is any reference to what pressures are tolerated by human plantar surfaces. These issues are beyond the scope of this presentation.

The relation between thickness and time as a result of a single loading cycle may be seen in Figure 13. Time-

related properties were determined by a static load tester. The tester consisted of a pivoted beam, jaws for the test sample, a pointer, and a scale. The sample was placed in the jaws and a weight was placed at the proper location on the beam to represent 10,20 , or 30 psi $(69,138$ or 207

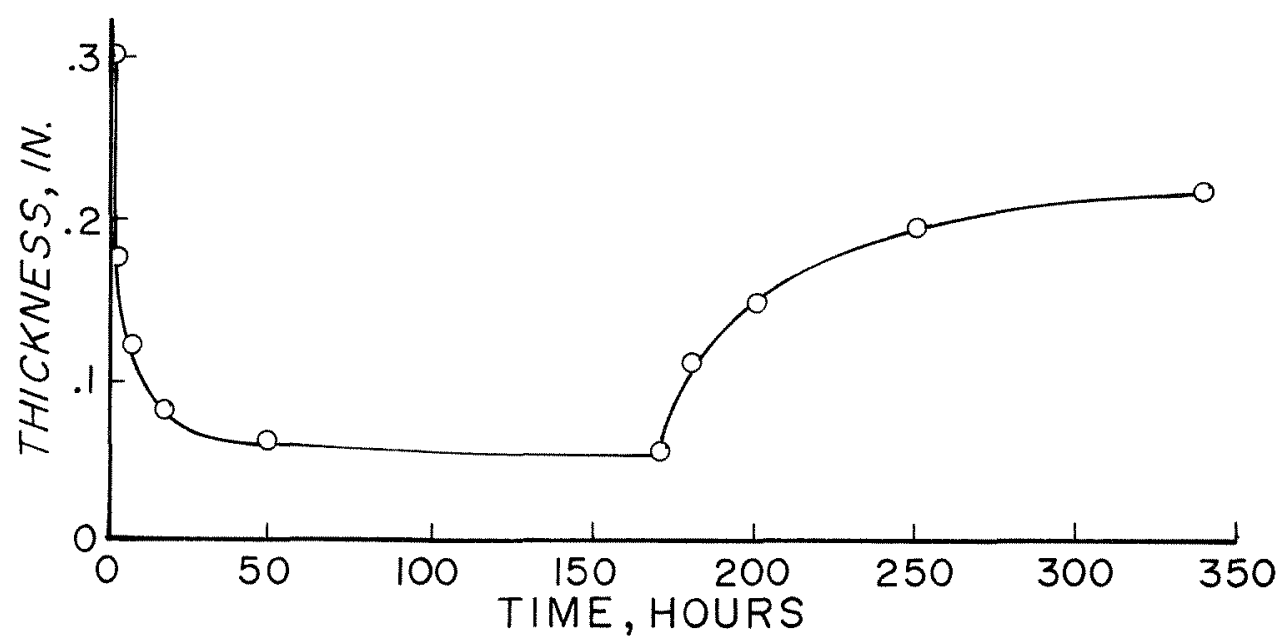

Figure 13.

Compression and recovery of medium density Plastazote. The static load was about $20 \mathrm{psi}(138 \mathrm{kPa})$ applied for one week. 


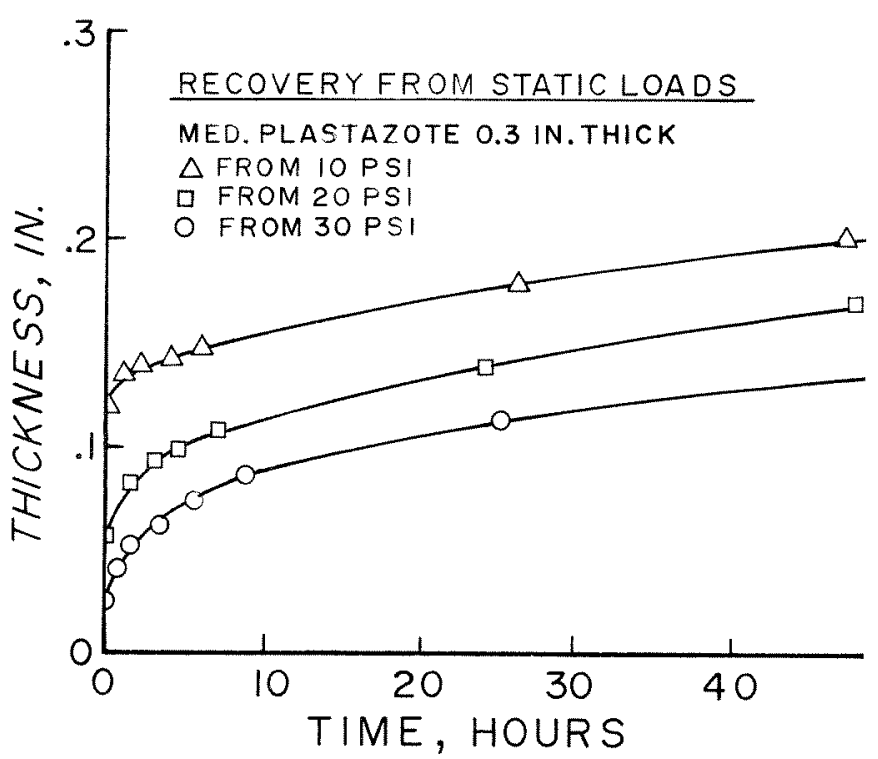

Figure 14.

Recovery of medium density Plastazote from different static loads applied for one week.

$\mathrm{kPa}$ applied pressure on the sample. The thickness of the sample was displayed on the scale. Illustrated is the effect of a static load of about 20 psi applied for one week on a sample of medium density Plastazote 0.3 inch $(0.76 \mathrm{~cm})$ thick. The material reached equilibrium at about one-sixth of the original thickness in about 100 hours. When the load was removed, the thickness reached equilibrium at about 77 percent of original thickness in about 180 hours. Static load recovery data for Plastazote at loads of 10,20 , and 30 psi are shown in Figure 14. The recovery thickness depends on the initial compression as determined by the compressive load. The effect of cyclic sustained loading may be seen in Figure 15. In this test, a one-half inch (1.27 $\mathrm{cm}$ ) thick sample of Aliplast $4 \mathrm{E}$ was cyclically loaded with approximately $30 \mathrm{psi}$. The load was applied in the morning and removed 8 hours later. Measurements of thickness were noted only at times of change of load. The thickness was recorded at 240 hours, approximately 130 hours after the conclusion of cyclic loading. The material had recovered to three-eighths of an inch $(0.95 \mathrm{~cm})$, or about 75 percent of original thickness. Cell window permeability accounts for this time-related behavior of the foam sample. As pointed out earlier, a sustained compressive load can squeeze out some of the gases from the cells. Upon removal of the load, the thickness will recover to varying extents depending upon the degree of mechanical integrity of the cellular structure.

In summary, these three examples of the time-related characteristics of closed cell plastic foams demonstrate the following: 1) during sustained loading, gas is forced out of the cells and more of the load is supported by elastic deformation of the cellular matrix; 2) when the load is removed, the potential energy stored in the plastic matrix

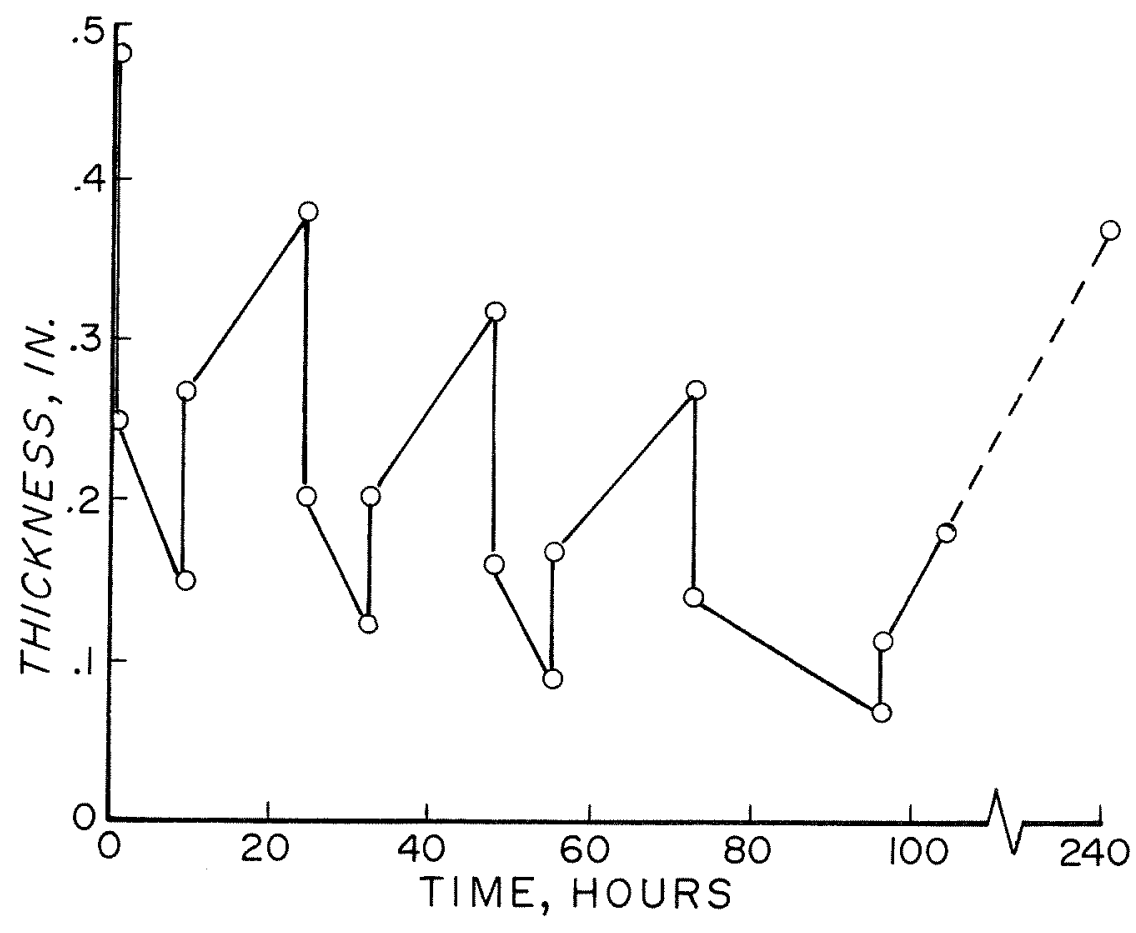

Figure 15.

Cyclic static loading of Aliplast 4E. Load was approximately $30 \mathrm{psi}(207 \mathrm{kPa})$. 
Journal of Rehabilitation Research and Development Vol. 27 No. 3 Summer 1990

starts drawing air inward in order to refill the cells; and, 3) as the spring strength decreases with increasing thickness, the recovery rate decreases.

\section{CONCLUSIONS}

In the clinical setting, awareness of the previously discussed factors have allowed us to select ways to provide appropriate foot/insole interface materials and to maintain their use over prolonged periods of time. For example, many of the patients we encounter in our clinical study tend to obesity (i.e., 200 to 300 pounds, or 441 to $661 \mathrm{~kg}$ ). In order to reduce the otherwise high plantar pressures, our results suggest the use of thicker interface materials. Moreover, an insole rotation procedure was instituted as a result of observed foam recovery properties. This procedure consisted of issuing several pairs of insoles for daily changes in order to permit insoles compressed during a day's use sufficient time to recover in thickness during the interval of nonuse.

In conclusion, the practical implications of these illustrations and comments are to increase the awareness of factors important in choices of available materials for applications such as insoles for footwear. Materials are available in a variety of cell structures, compliance, compressibility, and thickness. These foam characteristics should be considered in the treatment of patients of different weights and varied contours of the plantar surface. Our observations of the time-related and nontime-related phenomena suggest an avenue of improved care for the patient requiring insoles for footwear. Issuing thicker insoles offers a reduction of pressure concentration experienced by certain categories of patients. Moreover, issuing several pairs of insoles for daily rotation allows a recovery period during the nonwear interval to offer better cushioning during a day of wear.

\section{GLOSSARY}

Adiabatic. A change in volume or pressure without a change in thermal energy.

Bubbles. Referring to cells in a cellular foam.
Cell size. Indicating the relative size of cells or bubbles which compose a cellular foam.

Cell wall. Structural component separating two cells; also known as cell window.

Cellular foam. A mass of bubbles composed of a plastic and a gas phase.

Closed cell. Discrete cell through which gases do not pass freely.

Compliance factor $(a)$. Characterizes the rapidity at which thickness changes with load in compression.

Compressibility factor $(r)$. Characterizes the fraction of the unloaded thickness at which a limit is approached in compression.

Compressive load. Normal force per unit area in compression.

Density. Mass of polymer material per unit volume of the material.

Hysteresis. Failure of related phenomena to keep pace with each other (i.e., the delay in recovery of thickness upon removal of load under conditions of rapid loading).

Nonreticulated. Structure with intact walls separating the cells.

Nontime-related. Taking place under rapid cyclic loading conditions as may occur during a gait cycle.

Open cell. Discrete cell with open cell window through which gases may pass freely.

Potential energy. Energy stored in the cellular matrix as a result of compression.

Pressure (p). Normal force per unit area.

Reticulated. Structure with openings in cell walls.

Rib. Structural component of the cell occurring at the line of cell intersections which is capable of resisting a compressive load; also known as strand.

Time-related. Occurring under sustained loading conditions as in static loading or extended cyclic loading.

Work (w). Addition of energy through compression (i.e., change in volume as a result of applied pressure).

\section{REFERENCES}

1. Blair EA: Cell structure: Physical property relationships in elastomeric foams. Cellular Plastics, Washington, DC: National Academy of Sciences, 143-152, 1967.

2. Skochdopole RE, Rubens LC: Physical property modifications of low-density polyethylene foams. J Cell Plastics 1:91-96, 1965. 